\title{
Impact of the Sun on Remote Sensing of Sea Surface Salinity from Space
}

\author{
David M. Le Vine ${ }^{1}$, Saji Abraham ${ }^{1}$, F. Wentz ${ }^{2}$, G.S.E. Lagerloef ${ }^{3}$ \\ (1) Goddard Space Flight Center, Greenbelt, MD 20771 \\ email: David.M.LeVine@nasa.gov \\ (2) Remote Sensing Systems, 438 First Street, Suite 200, Santa Rosa, CA 95401 \\ email: Frank.Wentz@remss.com \\ (3) Earth \& Space Research, 1910 Fairview Ave E, Suite 210 Seattle, WA 98102 \\ email: lager@esr.org
}

\begin{abstract}
The sun is a sufficiently strong source of radiation at L-band to be an important source of interference for radiometers on future satellite missions such as SMOS, Aquarius, and Hydros designed to monitor soil moisture and sea surface salinity. Radiation from the sun can impact passive remote sensing systems in several ways, including line-of-sight radiation that comes directly from the sun and enters through antenna side lobes and radiation that is reflected from the surface to the radiometer. Examples are presented in the case of Aquarius, a pushbroom radiometer with three beams designed to monitor sea surface salinity. Near solar minimum, solar contamination is not a problem unless the sun enters near the main beam. But near solar maximum, contamination from the sun equivalent to a change of salinity on the order of 0.1 psu can occur even when the signal enters in sidelobes far from the main beam.

Keywords-Ocean Salinity, microwave radiometry; remote sensing;
\end{abstract}

\section{INTRODUCTION}

The solar radio flux, $F_{\lambda}$, is the brightness at wavelength $\lambda$ integrated over the entire solar disc. Since the solar disc is small this is approximately:

$$
\mathrm{F}_{\lambda}=\mathrm{B}_{\text {sun }} \Omega_{\text {sun }}
$$

where the mean brightness, $\mathrm{B}_{\text {sun }}$, is expressed in $\mathrm{Wm}^{-2} \mathrm{~Hz}^{-1} \mathrm{sr}^{-1}$ and $\Omega_{\text {sun }}$ is the solid angle (steradians) of the sun as seen from the earth: $\Omega_{\text {sun }}=2 \pi\left[1-\cos \left(\theta_{\text {sun }}\right)\right]=8.2210^{-5}$. In this expression $\theta_{\text {sun }}$ is the angular radius of the sun as viewed from the earth. At $1.4 \mathrm{GHz}, \theta_{\text {sun }}=0.293$ degrees which is about $10 \%$ greater than the optical angular radius [1].

The solar flux, $\mathrm{F}_{\lambda}$, is typically expressed in solar flux units, $s f u$ $\left(1 \mathrm{sfu}=10^{-22} \mathrm{Wm}^{-2} \mathrm{~Hz}^{-1}\right)$. Values at various wavelengths are available daily from ground stations around the world that monitor the sun. Among the stations monitoring radiation at $1.415 \mathrm{GHz}$ are the observatories in the Radio Solar Telescope Network (RSTN) operated by the U.S. Air Weather Service (Sagamore Hill, 42 N, 71 W; Learmonth, Australia 22 S, 114 E; Palehua, Hawaii 21N, 158W; and San Vito, Italy 41 N, 18 E) whose data can be obtained through the National Geophysical Data Center at Boulder, Colorado [2].
Solar flux units can be converted into an equivalent blackbody temperature by assuming that the sun is a uniform thermal source (blackbody) and using the Rayleigh-Jeans approximation. The relationship between the equivalent temperature, $\mathrm{T}_{\text {sun }}$, and the solar flux $\mathrm{F}_{\lambda}$ can be written:

$$
\mathrm{T}_{\text {sun }}=\left[\lambda^{2} / 2 \mathrm{k}\right] \mathrm{B}_{\text {sun }}=\left[\lambda^{2} / 2 \mathrm{k} \Omega_{\text {sun }}\right] \mathrm{F}_{\lambda} 10^{-22}
$$

At L-band (1.4 GHz), one obtains:

$$
\mathrm{T}_{\text {sun }} \approx 2000 \mathrm{~F}_{\mathrm{L}}
$$

where $F_{L}$ is the solar flux at L-band in solar flux units (sfu).

\section{EXAMPLES}

It is convenient to separate radio emission from the active sun into two categories on the basis of their characteristic scale of temporal variation [3]:

$$
\mathrm{T}_{\text {sun }}(\mathrm{t})=\mathrm{T}_{\mathrm{S}}(\mathrm{t})+\mathrm{T}_{\mathrm{R}}(\mathrm{t})
$$

$\mathrm{T}_{\mathrm{S}}$ represents a slowly varying component (time scale of hours to days) and at L-band is largely due to thermal radiation from the hot plasma in the atmosphere of the Sun. It varies randomly from day to day with a mean value and standard deviation that vary with the 11-year solar cycle and correlate with the number of sunspots. Figure 1 shows the $T_{S}$ component of the solar flux at $1.4 \mathrm{GHz}$ measured at the Sagamore Hill solar observatory [2]. The figure shows the daily value of flux measured at local noon 1980 through 2004. Figure 2 shows similar data for a period of one year with higher temporal resolution. The blue curve is for the year, 1986, a minimum in the solar cycle and the red curve is for 1989 , which is near the maximum of the same solar cycle.

$T_{R}$ is a rapidly varying component (seconds to minutes in duration) associated with flares (explosions on the surface of sun) and other transient activity on the sun. Solar flares occur due to a sudden release of magnetic energy in the solar atmosphere and their frequency varies with the eleven-year solar cycle. During periods of low solar activity few solar 


\section{Report Documentation Page}

Form Approved

OMB No. 0704-0188

Public reporting burden for the collection of information is estimated to average 1 hour per response, including the time for reviewing instructions, searching existing data sources, gathering and maintaining the data needed, and completing and reviewing the collection of information. Send comments regarding this burden estimate or any other aspect of this collection of information,

including suggestions for reducing this burden, to Washington Headquarters Services, Directorate for Information Operations and Reports, 1215 Jefferson Davis Highway, Suite 1204, Arlington

VA 22202-4302. Respondents should be aware that notwithstanding any other provision of law, no person shall be subject to a penalty for failing to comply with a collection of information if it

does not display a currently valid OMB control number.

\begin{tabular}{|c|c|c|}
\hline $\begin{array}{l}\text { 1. REPORT DATE } \\
\mathbf{2 5} \text { JUL } 2005\end{array}$ & $\begin{array}{l}\text { 2. REPORT TYPE } \\
\text { N/A }\end{array}$ & $\begin{array}{l}\text { 3. DATES COVERED } \\
\text { - }\end{array}$ \\
\hline \multirow{3}{*}{\multicolumn{2}{|c|}{ Impact of the Sun on Remote Sensing of Sea Surface Salinity from Space }} & 5a. CONTRACT NUMBER \\
\hline & & 5b. GRANT NUMBER \\
\hline & & 5c. PROGRAM ELEMENT NUMBER \\
\hline \multirow{3}{*}{\multicolumn{2}{|c|}{ 6. AUTHOR(S) }} & 5d. PROJECT NUMBER \\
\hline & & 5e. TASK NUMBER \\
\hline & & 5f. WORK UNIT NUMBER \\
\hline \multicolumn{2}{|c|}{$\begin{array}{l}\text { 7. PERFORMING ORGANIZATION NAME(S) AND ADDRESS(ES) } \\
\text { Goddard Space Flight Center, Greenbelt, MD } 20771\end{array}$} & $\begin{array}{l}\text { 8. PERFORMING ORGANIZATION } \\
\text { REPORT NUMBER }\end{array}$ \\
\hline \multirow{2}{*}{\multicolumn{2}{|c|}{ 9. SPONSORING/MONITORING AGENCY NAME(S) AND ADDRESS(ES) }} & 10. SPONSOR/MONITOR'S ACRONYM(S) \\
\hline & & $\begin{array}{l}\text { 11. SPONSOR/MONITOR'S REPORT } \\
\text { NUMBER(S) }\end{array}$ \\
\hline
\end{tabular}

12. DISTRIBUTION/AVAILABILITY STATEMENT

Approved for public release, distribution unlimited

13. SUPPLEMENTARY NOTES

See also ADM001850, 2005 IEEE International Geoscience and Remote Sensing Symposium Proceedings (25th) (IGARSS 2005) Held in Seoul, Korea on 25-29 July 2005. , The original document contains color images.

14. ABSTRACT

15. SUBJECT TERMS

16. SECURITY CLASSIFICATION OF:

a. REPORT

unclassified b. ABSTRACT

unclassified c. THIS PAGE

unclassified
17. LIMITATION OF ABSTRACT

UU
18. NUMBER OF PAGES

4 19a. NAME OF

RESPONSIBLE PERSON 
flares are detected and there can be thousands during periods of high solar activity. Not all flares are associated with an enhancement of radiation at microwave frequencies. When such enhancements occur they are called Solar Microwave Bursts (SMB). A worldwide network of observing stations report the SMB at variable frequencies and thresholds. The Radio Solar Telescope Network (RSTN) reports bursts when the flux exceeds $50 \mathrm{sfu}$. The Owens Valley Solar Array (OVSA) records solar flares with lower thresholds by measuring changes from RMS fluctuations over quiet periods before the bursts [4].

Figure 3 provides information about the distribution of peak amplitude in solar flux units (top) and the distribution of duration in minutes (bottom) of radiation at L-band during SMB. The data are from RSTN observations at $1.4 \mathrm{GHz}$ during 1989, a period of high solar activity. Each bar represents the number of events in a box centered on its midvalue and $50 \mathrm{sfu}$ wide. The boxes in the histogram on the right are 5 minutes wide and also centered on its mid-value. The last bar in each case represents the remainder. The first bar in the histogram of peak amplitudes is an under estimate because of the $50 \mathrm{sfu}$ threshold adopted by the RSTN. The occurrences in this box were identified because of recordings at other frequencies (i.e. the threshold exceeded $50 \mathrm{sfu}$ at a frequency other than $1.4 \mathrm{GHz}$ ). The peak flux can differ for the same flare recorded at different stations. In such cases, the recording with the largest peak value (at $1.4 \mathrm{GHz}$ ) was used.

\section{DIRECT AND REFLECTED RAY}

Radiation from the sun can impact remote sensing of the ocean surface from space in several ways: (a) Radiation can enter the antenna via the "direct ray" along the line-of-sight from sun to spacecraft; (b) The mean (average) signal reflected from the surface behaves like a ray that is specularly reflected from the ocean surface (this "reflected ray" can be nearly as strong as the direct ray); and (c) The fluctuating portion of the reflected signal (incoherent signal) that is due to the wave roughened surface and often referred to as "glitter". Only the first two terms will be discussed here.

The contribution to the antenna temperature due radiation that comes directly (line-of-sight) from the sun can be obtained in the conventional manner by integrating the antenna over the solar disk. Using the Rayleigh-Jeans approximation to express the solar brightness in terms of equivalent temperature, one obtains:

$$
\mathrm{T}_{\mathrm{A}}=\left(1 / \Omega_{\mathrm{p}}\right) \int \mathrm{T}_{\text {sun }}(\Omega) \mathrm{F}_{\mathrm{n}}(\Omega) \mathrm{d} \Omega
$$

where $\Omega_{p}$ is the beam solid angle and $F_{n}$ is the normalized antenna pattern. If $\mathrm{T}_{\text {sun }}$ is constant and the range of integration small, then to a good first approximation:

$$
\mathrm{T}_{\mathrm{A}}=\left(1 / \Omega_{\mathrm{p}}\right) \mathrm{T}_{\text {sun }} \mathrm{F}_{\mathrm{n}} \Omega_{\text {sun }}
$$

Substituting for $\mathrm{T}_{\text {sun }}$ from Equation (2) and introducing the antenna (directional) gain $G=\left(4 \pi / \Omega_{p}\right) F_{n}$, one obtains

$$
\begin{aligned}
\mathrm{T}_{\mathrm{A}} & =\left(1 / \Omega_{\mathrm{p}}\right)\left[\lambda^{2} / 2 \mathrm{k}\right] \mathrm{F}_{\mathrm{n}} \mathrm{F}_{\mathrm{L}} 10^{-22} \\
& =0.013 \mathrm{GF}_{\mathrm{L}}
\end{aligned}
$$

Since radiation from the sun is largely unpolarized $G$ should be interpreted as the sum of the co- and cross-polarized components.

The effect of the reflected ray can be computed in the same manner. The location of the specular point and angle of the incident radiation can be computed analytically for a spherical earth as described in [5]. Except for the fact that the reflected ray encounters the antenna at a slightly different angle, computation of the antenna temperature is the same as for the direct ray (Equations 5-7) but with gain in the appropriate direction. When reflection is from the ocean, the amplitude is comparable (reflectivity on the order of 0.7 ) but there will be differences in polarization because of the reflection coefficient at the surface is polarization dependent. In the case of land, the reflectivity is smaller (on the order of 0.3) and as a result the impact on antenna temperature will be somewhat smaller. As mentioned above, there will also be a contribution from the "incoherent" radiation (glint) which is not included here (and except in cases of large surface roughness will be smaller).

\section{IMPACT ON AQUARIUS}

As an illustration of the potential impact on remote sensing at L-band, calculations are presented for configuration of the Aquarius mission. Aquarius is 3 beam pushbroom radiometer designed to map sea surface salinity on a monthly basis with an accuracy of $0.2 \mathrm{psu}$. The sensor will be in a $6 \mathrm{am} / 6 \mathrm{pm}$ sunsynchronous orbit with the main beams pointing toward the nighttime side of the terminator (looking away from the sun). Hence, both the direct ray and the ray reflected from the surface will enter through antenna sidelobes far from the main beam. The antenna consists of a $2.5 \mathrm{~m}$ offset fed parabola with three feeds. Figure 4 illustrates the antenna pattern for one of the beams. The figure shows a cut through the pattern at constant azimuth ( $\varphi=175$ degrees $)$ as a function of polar angle ( $\theta$ in spherical coordinates). Positive $\theta$ corresponds to $0<\varphi<\pi$ and negative $\theta$ corresponds to the $\pi<\varphi<2 \pi$. The green curve is the cross-pol term and the red curve is the sum of coand cross-pol. These are theoretical curves obtained numerically for the present design and ignore the presence of the spacecraft. Figure 5 shows where the direct and reflected ray appear. The locations are for an idealized case (spherical earth and circular orbit with sun fixed in position during the orbit) but give an idea of how the sun moves relative to the antenna as the sensor revolves in its orbit. At the top of Figure 5 are shown the locus of the direct ray (solid) and reflected ray (dashed) in the reference frame of the satellite. The sun is in the direction of the $y$-axis $(\varphi=90 \mathrm{deg})$ and the satellite orbits in the $\mathrm{z}$-x plane moving in the $\mathrm{x}$-direction. The loci of the two 
rays from the sun in the coordinates of the antenna are plotted at the bottom of the figure ( $\mathrm{Z}$ is in the boresight direction, $\mathrm{X}$ in the direction of motion of the spacecraft and polar coordinates $(\theta, \varphi)$ are measured with respect to these Cartesian coordinates in the usual manner). Figure 5 is a polar plot in which theta is plotted radially in degrees and phi goes around the plot (0360). The loci change seasonally as the solar angle changes with respect to the plane of the earth's rotation. They reduce to a point at the equinox and grow to maxima (not much different than shown in this example) at the solstice. At slightly large solar angles one begins see a shadow region as the earth blocks rays from the sun (reflected and direct ray).

Figure 6 shows the effect of the sun on the antenna temperature as the satellite orbits for one full year (the sun and satellite go through the full range of relative orientation). The sum of the direct and reflected ray is plotted. The vertical axis is latitude. The ascending orbit is at the bottom half of the figure and the descending orbit at the top. The dark horizontal line at the center represents the North Pole. The data are averaged for one day (14 orbits) and then results are plotted for each day (365 vertical stripes along horizontal axis). The calculation has been done for a constant solar flux of $250 \mathrm{sfu}$ and uses the antenna shown in Figure 4 pointing at 33.8 degrees. (This is the middle of the three Aquarius beams and is the angle the boresight ray makes with nadir at the spacecraft.) At the top of Figure 6 is the result for vertical polarization and the bottom panel in Figure 6 shows the result for horizontal polarization. The contribution of the sun at $\mathrm{V}$ pol is a little less than at $\mathrm{H}$-pol because the reflection coefficient is smaller at $\mathrm{V}$-pol than at H-pol for the reflected ray. The patterns in the figures are caused by the motion of the sun relative to the antenna pattern as the satellite orbits and motion of the sun relative to the orbit during the course of the year. Because the sun is relatively small, the changes as it moves relative to the peaks and valleys of the sidelobes cause the patterns noticeable in Figure 6. In Figure 6 land has the same reflectivity as ocean (so, in this sense it is a worst case).

\section{CONCLUSIONS}

The goal for the Aquarius mission is a monthly map with an accuracy of $0.2 \mathrm{psu}$. In radiometric terms, a change of $1 \mathrm{psu}$ corresponds roughly to a change in brightness temperature of about $0.5 \mathrm{~K}$ (the sensitivity depends on salinity, temperature, polarization and incidence angle). Hence, the goal in radiometric terms is on the order of $0.1 \mathrm{~K}$.

From Figure 6 it is clear that in most cases the contribution from the sun is on the order of $0.05 \mathrm{~K}$ or less. But it can be as much as the threshold of $0.1 \mathrm{~K}$. In this sense, the sun is clearly a concern for remote sensing of salinity. On the other hand, a solar flux of $250 \mathrm{sfu}$ is a rather large value for the $T_{S}$ component and is likely to be encountered only during solar maximum and then only occasionally (i.e. Figure 1). At solar minimum $\left(\mathrm{F}_{\mathrm{L}}=50\right)$, contamination from the sun does not present a major problem. Nevertheless, the contamination by the sun is only one component of many that make up the error budget of the sensor, and to accurately assess the impact one must include all the factors together.

Figure 6 shows only the $\mathrm{T}_{\mathrm{S}}$ component. The contribution from solar flares can be much larger. As shown in Figure 3, one can expect a significant number of flares with $\mathrm{F}_{\mathrm{L}}$ exceeding 500 sfu during the course of a year during solar maximum. However, the duration is relatively brief. The total duration of flares (area under the distribution in Figure 3) is less than 4\% of the total time. It will be even less for conditions other than solar maximum. Hence, a reasonable approach might be simply to flag the occurrence of flares and then evaluate the impact based on the reported magnitude. Data from a global monitoring network such as the RSTN can be used a posteriori to determine if the flare was large enough to exceed the threshold of tolerance set by the side lobe level. The lost data should be even less than $4 \%$ because most flares have peak flux levels below $200 \mathrm{sfu}$.

\section{REFERENCES}

[1]. Aarons, J. Solar System Radio Astronomy, Plenum, 1965.

[2]. http://www.ngdc.noaa.gov/stp/SOLAR/ftpsolarradio.html

[3]. Kraus, J.D., Radio Astronomy, McGraw-Hill, 1966, Chapter 8, Section 7.

[4]. http://www.ovsa.njit.edu/

[5]. Le Vine, D.M. and S. Abraham, "Galactic Noise and Passive Microwave Remote Sensing from Space at L-Band," IEEE Trans. GRS, Vol 42 (\#1), pp. 119-129, 2004.

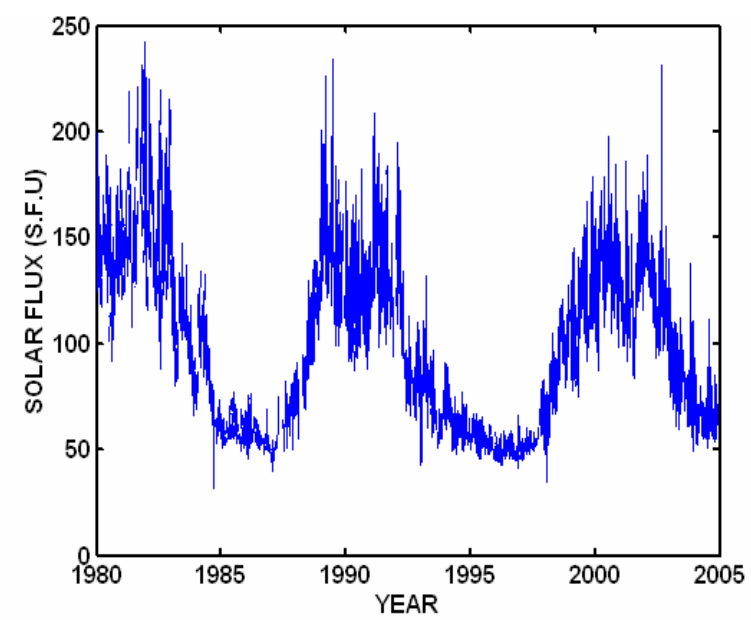

Figure 1. Solar flux at $1.4 \mathrm{GHz}$ at noon from the Sagamore Hill [2].

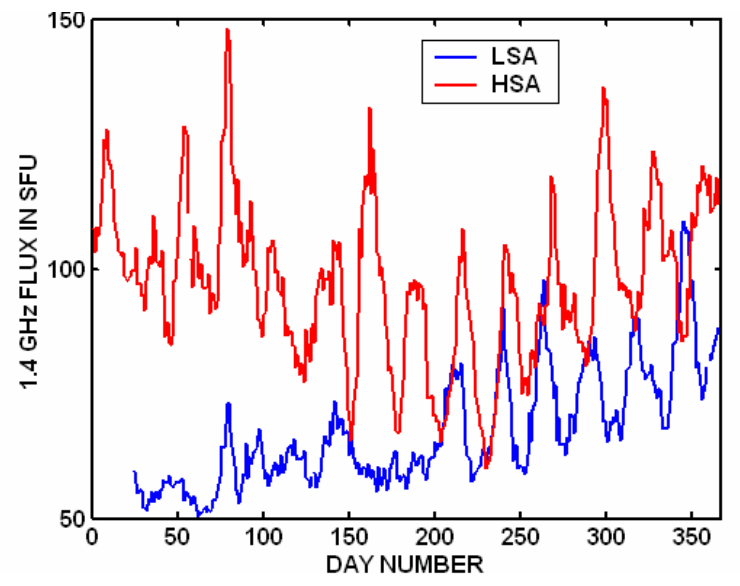

Figure 2. Solar flux at $1.4 \mathrm{GHz}$ at noon. High solar activity (1989, red) and low solar activity (1986, blue). Data are from Sagamore Hill [2]. 

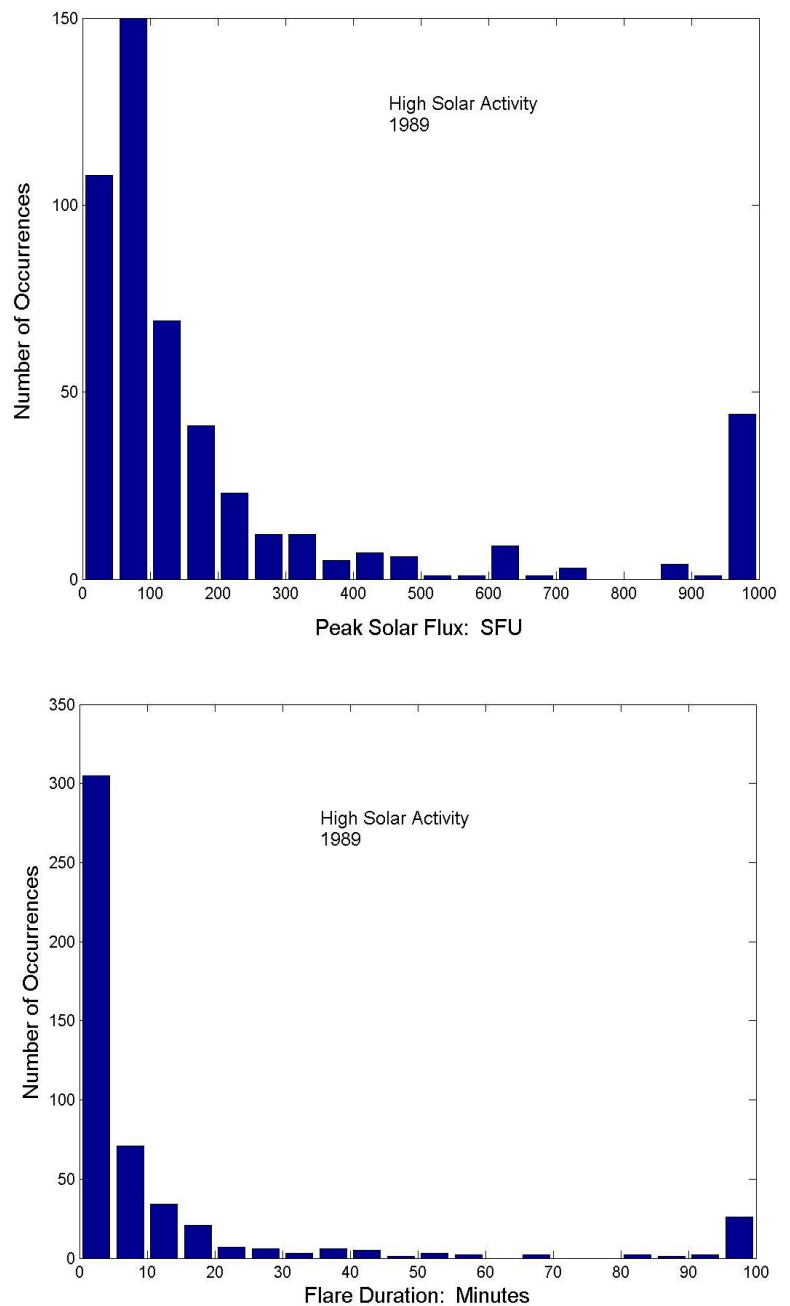

Figure 3. Histograms showing distribution of peak amplitude (top) and duration (bottom) for SMB at $1.4 \mathrm{GHz}$ during high solar activity. The data are from the RSTN during 1989.

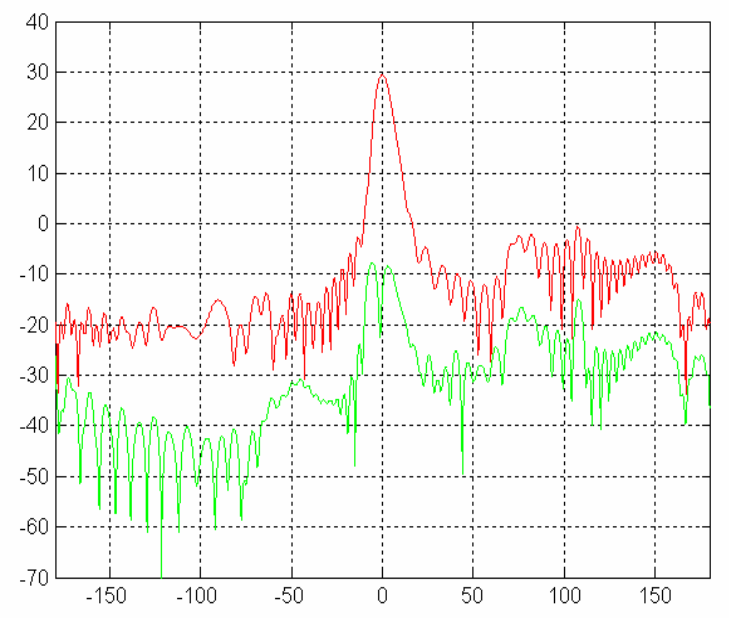

Figure 4. Antenna gain of an antenna similar to that planed for Aquarius. Green is the cross-polarized component and red the sum of cross- and co-polarized gain.

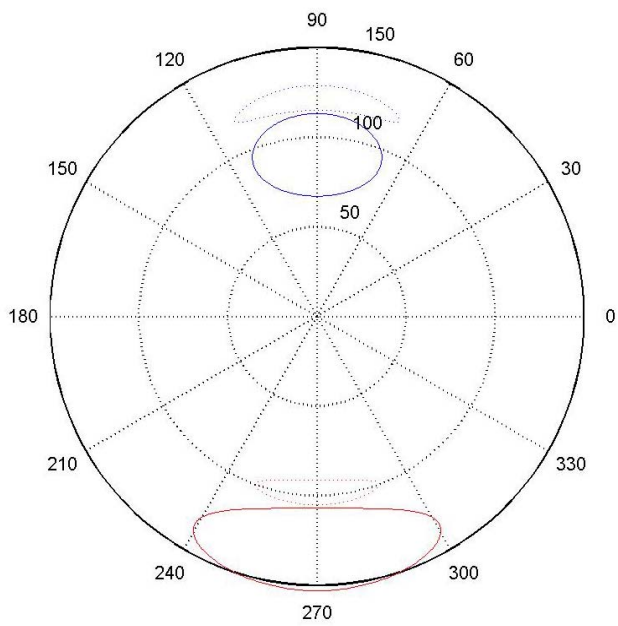

Figure 5. Polar plot showing the loci of location of direct (solid) and reflected (dashed) rays as the satellite revolves in its orbit. At the top are the loci in coordinates fixed on the spacecraft and on the bottom are same in antenna coordinates. Note that $\pi<\varphi<2 \pi$ corresponds to $\theta<0$ in Fig 4 .
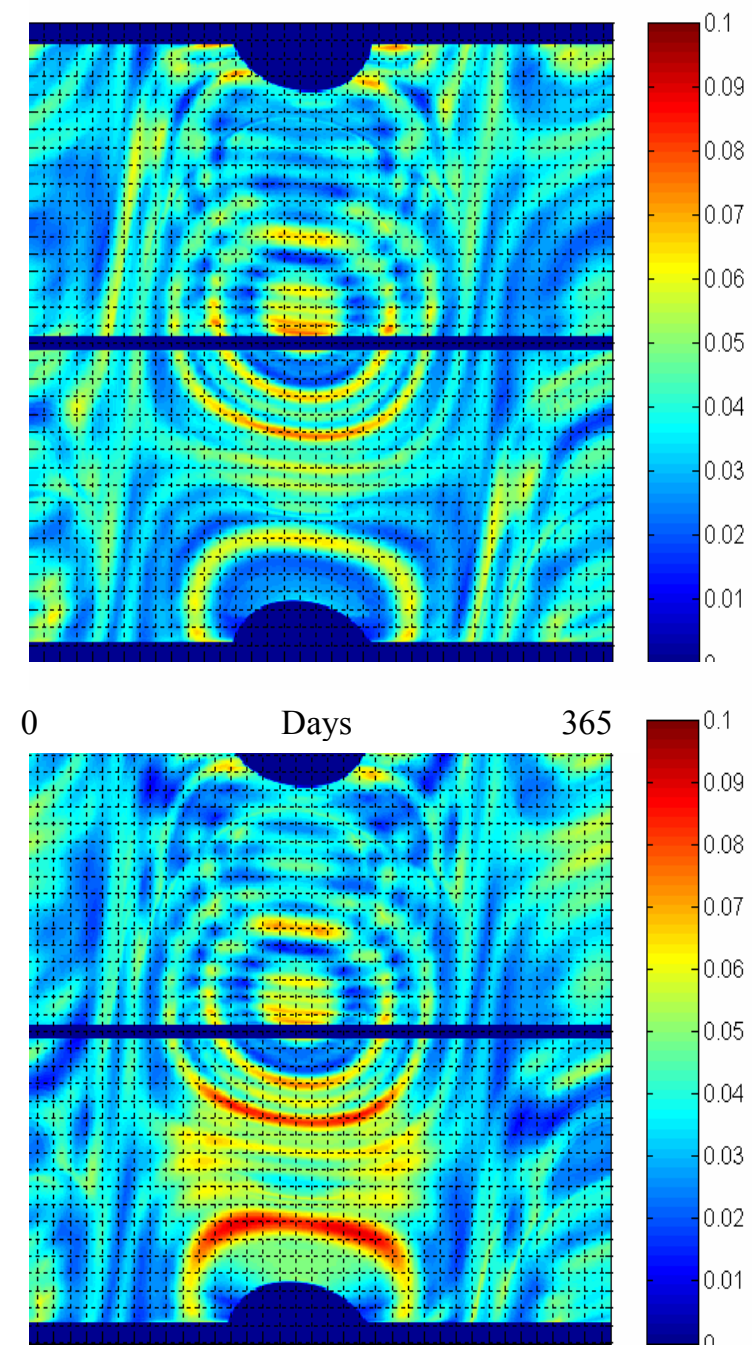

Figure 6. Antenna temperature due to the sum of direct and reflected ray for vertical polarization (top) and horizontal polarization (bottom) for one year. Color code is in Kelvin. 